\title{
La femme masculinisée dans la presse mondaine française de la Belle Époque
}

The Masculinized Woman in the French Press of the "Belle Epoque"

\section{Guillaume Pinson}

\section{(2) OpenEdition}

1 Journals

Édition électronique

URL : http://journals.openedition.org/clio/9471

DOI : 10.4000/clio.9471

ISSN : 1777-5299

Éditeur

Belin

Édition imprimée

Date de publication : 15 décembre 2009

Pagination : 211-230

ISSN : 1252-7017

Référence électronique

Guillaume Pinson, "La femme masculinisée dans la presse mondaine française de la Belle Époque »,

Clio. Histoire, femmes et sociétés [En ligne], 30 | 2009, mis en ligne le 15 décembre 2012, consulté le 02 mai 2019. URL : http://journals.openedition.org/clio/9471 ; DOI : 10.4000/clio.9471

Tous droits réservés 


\section{La femme masculinisée dans la presse mondaine française de la Belle Époque}

Guillaume PINSON

Au XIXe siècle, la modernité médiatique s'est largement construite sur un imaginaire de la femme fondée en nature et non en culture ${ }^{1}$. Généralement, on connaît mieux les avatars littéraires de cet imaginaire $^{2}$, mais le journal, la presse en général, ont sans doute fait plus encore pour structurer les catégories mentales, les perceptions et les pratiques «genrées » des lecteurs et des lectrices. Tout au long du siècle, suivant une logique qui va longtemps perdurer et perdure encore dans bien des cas, le journal est soigneusement organisé en espaces cloisonnés, les uns destinés aux lecteurs, les autres aux lectrices $^{3}$. Même s'ils sont considérables, il est toutefois difficile de mesurer avec précision les effets sociaux de ces compartimentations du journal, et sans doute ne faut-il pas non plus les exagérer tant les échanges et les porosités ont été nombreux à l'intérieur même du journal. Lorsqu'on s'intéresse à la presse mondaine à la Belle Époque - comme je me propose de le faire dans cet article, et pour prolonger ce que j'ai développé ailleurs ${ }^{4}$, il s'avère difficile, à partir des textes mêmes, de trancher avec certitude sur le type de lecteurs visés, hommes ou femmes, et encore plus sur les lecteurs ou lectrices effectifs et leurs pratiques de lectures réelles.

Il s'agit donc de considérer le délicat - et traditionnel - problème de la correspondance entre les injonctions textuelles et la consommation tangible de l'imprimé. Or, quoique largement

\footnotetext{
Angenot 1989 : 478.

Planté 1989.

Eveno 2003.

Pinson 2008.
} 
féminine, accompagnant le vieux stéréotype d'une mondanité organisée autour de "la femme», la presse mondaine se développe aussi à l'intersection de plusieurs mandats: la représentation des élites, du temps des loisirs, de la mode, des sports, des divertissements, croisant même, un cran hiérarchique plus bas dans le discours social, tout le secteur «faisandé » de la presse humoristique, coquine et "pornographique»-comme on le dit à l'époque pour qualifier une presse licencieuse mais peu audacieuse selon les standards actuels. Elle est marquée également par la nouvelle vitalité d'une presse féminine au début du $\mathrm{XX}^{\mathrm{e}}$ siècle. Il faut se garder de situer ce vaste secteur médiatique à l'intérieur de pratiques de lecture et de représentations uniquement féminines. Certains quotidiens de la haute bourgeoisie ou de l'aristocratie, comme Le Figaro et Le Gaulois par exemple, font une place à la mondanité dans l'espace du périodique - c'est le cas du carnet mondain - et captent certainement, au cœur de publications avant tout destinées à un lectorat masculin, l'attention régulière de femmes très engagées dans les réseaux sociaux de la capitale. Certaines sources l'attestent, comme le journal intime de la marquise de Breteuil, rédigé en 1885 et 1886 et publié par Éric Mension-Rigau ${ }^{5}$ : la marquise, souvent tenue à l'écart des mondanités parce que de santé fragile, est pourtant très au fait des grandes manifestations mondaines parisiennes et même européennes grâce à la lecture des rubriques mondaines du Gaulois ou du Figaro. Mais son journal intime montre aussi que la marquise est loin de se cantonner à la lecture du carnet mondain. Elle lit avidement les nouvelles politiques et diplomatiques, qu'elle commente régulièrement. Autrement dit, sa pratique de lecture et les usages qu'elle fait de ces lectures ne respectent pas les injonctions habituelles ni les frontières tracées à l'intérieur même du quotidien.

Ainsi, sans nier certaines des spécificités des périodiques, telles la hiérarchisation sociale des titres et l'importance de la répartition sexuée de leur usage, la presse mondaine demeure encore dans le dernier quart du siècle une vaste nébuleuse de publications dont les limites et les appropriations ne sont pas aussi évidentes qu'on ne le pense habituellement. En outre, cette mouvance qui caractérise le

5 Castelbajac 2003. 
secteur des publications mondaines s'accompagne à la fin du XIX siècle d'un imaginaire anxiogène, celui de la «fin d'un sexe » et de la masculinisation de la femme ${ }^{6}$. Cette angoisse que la presse mondaine ne peut contenir est une forme de récupération des anxiétés contemporaines qui traversent l'ensemble du discours social. Elle contribue aussi à déstabiliser la division entre le féminin et le masculin à l'intérieur même de ce type de publication. L'inquiétude est liée à de réelles modifications dans les pratiques. On sent en effet que la presse mondaine et féminine est alors préoccupée par «la femme émancipée ", par ses nouveaux comportements sociaux, par certaines pratiques, comme les sports par exemple, qui déplacent certaines limites et conventions, en particulier dans l'aristocratie et la haute bourgeoisie qui l'imite 7 . Pour les journaux et magazines mondains et féminins, cette situation source de tâtonnement dans l'attitude à adopter face à des comportements perçus comme importés de la sphère masculine, exige une adaptation de leur présentation et de leur contenu.

Au début du $\mathrm{XX}^{\mathrm{e}}$ siècle, cette anxiété sociale s'estompe déjà quelque peu, ou du moins ne s'exprime plus de la même manière. La presse mondaine plus conservatrice, héritière de pratiques médiatiques qui peuvent remonter jusqu'au XVIII siècle, tend à s'effacer devant l'arrivée des magazines résolument modernes, tels que Femina (1901) et La Vie heureuse (1902), destinés à un lectorat féminin dont l'horizon des pratiques est en voie d'élargissement. Si la limite entre féminin et féminisme demeure assez ambiguë dans ces revues $^{8}$, la représentation des femmes et de pratiques naguères jugées viriles y est plus affirmée et livrée dans une tonalité moins crépusculaire. Originales, ces publications font notablement évoluer la représentation de la féminité. C'est cette période de transition, à travers l'image de la femme masculinisée et de ses avatars, que j'aimerais explorer dans cet article. Il s'agit de montrer notamment que le passage au travers d'une «crise de la masculinisation » a permis

\footnotetext{
6 Murat 2006 et Rochefort 1999.

7 Flamant-Paparatti 1984.

8 Comme l'a notamment montré Roberts 1997 à propos de La Fronde. Voir aussi Blum 2002.
} 
un renversement, celui d'une féminité sur la défensive à une féminité qui s'affirme, ne craignant plus de lier activités mondaines et représentation d'une femme pour ainsi dire « virilisée ».

\section{La « fin d'un sexe ", angoisses fin de siècle}

À la fin du XIX ${ }^{\mathrm{e}}$ siècle, la représentation de «la femme masculinisée » trahit une presse mondaine menottée par les stéréotypes à la fois contemporains et déjà anciens sur la place de « la femme» dans la société et par les anxiétés qui traversent alors le discours social. Tout un imaginaire que l'on attendrait mièvre et déproblématisé - la galanterie pédagogique sur les devoirs domestiques de "Madame», la mise en scène des activités de la sphère féminine, la mode... - est contaminé par le paradigme sombre de ce que Marc Angenot a appelé la «déterritorialisation symbolique », sentiment diffus mais généralisé d'un glissement des repères symboliques et sociaux ${ }^{9}$. Pour les chroniqueuses des revues mondaines, il y a là une posture tentante mais difficile à tenir, qui doit relier futilités mondaines et inquiétudes "sérieuses » sur le monde tel qu'il va. On voit donc proliférer dans les années 1880 et 1890 un discours idéologique assez appuyé, souvent livré sous la forme d'un autodénigrement féminin fondé sur une logique de l'intériorisation par les femmes de la «domination masculine $»^{10}$. Dans la Revue mondaine illustrée de 1892, par exemple, la chroniqueuse qui signe sous le pseudonyme de comtesse Jeanne Andrée abreuve ses lectrices d'une ironie amère sur la femme émancipée :

La question de la création d'un "Cercle de femmes» est revenue ces jours-ci dans les conversations et la presse a dû, de nouveau, s'en occuper.

Puisque vous voulez bien, mesdames, me faire la grâce de me lire, sinon de m'approuver, je vous dirai, sans plus de phrases, que cela ne peut être sérieux.

Consultez-vous, renseignez-vous ; auscultez votre âme, votre conscience, et vous me direz, après cela, si la création d'un Cercle de femmes vous paraît nécessaire.

\footnotetext{
$9 \quad$ Angenot 1989: 478.

10 Bourdieu 1998.
} 
Combien de vous, mariées, veuves ou petites filles, pourraient ou voudraient en faire partie?

Et puis, à quoi bon? Est-ce que nous ne les avons pas, nos cercles suffisamment exclusifs? Est-ce que nous ne nous rencontrons pas, toutes, où nous voulons nous rencontrer pour papoter tout à l'aise, sans crainte des indiscrétions ou des attentions, parfois agréables, mais assez souvent fatigantes, de ces messieurs : frères, époux, papas ou... amis ?

N'avons-nous pas nos respectifs five o'clock, nos promenades matinales, le pâtissier, le glacier, le parfumeur, le couturier, les sociétés de charité, les réunions pieuses, que sais-je ? Et j'en passe, et j'en passe (25 février 1892)!

Je ne m'attarderai pas sur ce discours récurrent des femmes qui font la leçon aux femmes. Pour les revues de la mondanité, il s'agit de tracer les contours d'une communauté « des femmes qui savent rester femme», nettement perceptible dans cet extrait par les adresses aux lectrices complices et par l'insistance d'un « nous » autant rassembleur qu'exclusif (« nos cercles», « nos five o'clock»...). La chroniqueuse de la Revue mondaine illustrée multiplie à l'envi ces rappels à l'ordre :

D]e me sépare, et avec empressement, des "apôtresses » de la féminité révolutionnaire quand elles émettent le désir de voir les femmes sortir du gynécée, du boudoir, du foyer pour concurrencer l'homme dans les diverses professions qu'on appelle libérales. Je ne me vois pas, - ni vous, Madame, - architecte, avocat, ingénieur ou médecin pas plus que je ne nous voie députées, sénatrices ou conseillères municipales!

$[\ldots]$

Laissez-nous donc ce que nous sommes [...] ; laissez-nous notre rôle, laissez-nous nous dévouer, laissez-nous sourire, laissez-nous pleurer, laissez-nous souffrir - cela nous plait ; - laissez-nous être l'éternelle blessée... si nous devons rester l' « éternelle aimée » (25 mai 1892).

La presse mondaine est un endroit hautement réactif à tout ce qui touche la place des femmes dans la société ; à la fin du siècle, celle-ci se caractérise par la fréquente représentation d'un «entre-soi menacé $»^{11}$, alors que la société française est agitée par les débats sur l'émancipation des femmes ${ }^{12}$. Ainsi, à travers l'image de la femme

11 Sur cette expression liée à la construction des identités sociales, voir Pinçon et Pinçon-Charlot 1995.

12 Voir Klejman \& Rochefort 1989, ainsi que Langlois 1979. 
masculinisée, le journal mondain se pose comme un lieu relativement instable où s'énoncent à la fois l'unité de la mondanité et la menace de sa dispersion.

Mais l'angoisse devant la femme virilisée va bien au-delà de la presse mondaine. Par effets de contiguité et de déplacement, c'est tout le secteur médiatique concernant les mondanités, les sports, la mode et les divertissements qui est aux prises avec cette inquiétude. Dans une publication comme La Vie parisienne, lancée en 1862, revue assez distinguée quoiqu'un peu plus égrillarde, le constat est amer, par exemple sous la plume d'un chroniqueur anonyme, le 25 janvier 1896 :

La femme, dans la société contemporaine, dans ce que les gazettes appellent le monde select et les épiciers le grand monde [...], la femme, qui devait être l'âme de cette élite, qui devait la diriger, lui donner le ton, lui imprimer son cachet et suppléer, par son tact et son influence, à ce que nos institutions et nos mœurs ont d'insuffisant et de funeste, a renoncé à son rôle naturel. Elle a abdiqué la suprématie mondaine, qui l'assujettissait, qui lui donnait un rôle actif et parfois difficile, pour conquérir ce qu'elle croit être son indépendance et pour vivre en garçon.

Ce ton rappelle celui d'un rédacteur des Causeries familières de 1889 qui résumait déjà le réquisitoire masculin contre les femmes masculinisées : «La Française de la fin de notre siècle a une tendance marquée à se masculiniser qui ne peut contribuer à l'embellir. Elle chasse, elle fume, elle affecte des allures indépendantes et provocantes; pour comble, enfin, elle demande à revêtir le costume masculin $»^{13}$. Et en effet tout le secteur de la mode est concerné par cette appréhension d'une masculinisation perçue comme une forme de dénaturation de la femme :

Il paraît que le sexe faible trouve que le règne du sexe fort a trop duré et, pour arriver à détrôner les hommes, les femmes n’auraient rien imaginé de mieux que d'unifier la mode, je veux dire de substituer les modes masculines aux modes féminines. (...) Le sexe faible va porter prochainement des pantalons. Il en est déjà aux petits chapeaux de feutres et aux cols droits; il déteste la féminité dans la mode (...). Je regardais l'autre jour au Bois, dans une voiture découverte, une jeune femme littéralement costumée comme un jeune homme: col cassé, chapeau de feutre rond, stick à la bouche. Or le premier mot que laissa

13 Causeries familières, 17, cité par Angenot 1989 : 485. 
échapper, près de moi, un brave homme de provincial qui n'était pas encore au courant des modes et toquades nouvelles, fut: "Quel est ce petit monster? $\gg^{14}$

Et le chroniqueur de L'Illustration d'ajouter :

Je gagerais qu'il y aura nombre de charmantes femmes ainsi masculinisées à Trouville, pendant cette grande semaine que voici - et ce sera dommage! Le diable emporte les fantaisistes et les exaspérées qui ont décidé de ne point se contenter de la beauté que la nature leur donne!

Puisque la femme est considérée comme fondée en nature et non en culture, sa masculinisation est une forme de dénaturation. Or celleci a pour résultat le règne effarant et incompréhensible de la laideur, et provoque des débats esthétiques. On voit ici ressortir toutes les contradictions d'un discours idéologique qui martèle sans cesse la «nature» de la femme mais qui est en réalité fondé et construit socialement, et par là esthétiquement, ce qui en fait la force. Comme l'écrit Michelle Perrot à propos de la mode vestimentaire, «l'esthétique est une violence aussi $»^{15}$. D'où les reproches bien connus qui seront inlassablement adressés à la femme à bicyclette, ou encore, un peu moins connus, qui concernent la femme en automobile.

L'automobile est intéressante à cet égard car elle est l'un de ces objets modernes qui, vers 1900, provoque des sentiments contradictoires, entre fascination et répulsion, comme la bicyclette dix ans plus tôt ${ }^{16}$. Tout le débat sur la femme en automobile se joue autour d'une question d'esthétique et provoque, chez les mondains, perplexité et angoisse : comment la femme pourrait-elle rester belle sur ces engins? «L'automobile est, si l'on peut dire, une sorte d'aérolithe qui tombe sans prévenir dans la mondanité, l'une de ces choses informes, sans pratique et sans avenir semble-t-il, qui fondent la laideur $»^{17}$. "J'ai peur que les automobiles, qui ont leur danger, ne

14 Rastignac, «Courrier de Paris », L'Illustration, 13 août 1887, cité par FlamantPaparatti $1984: 127$.

15 Perrot $1998: 377$.

16 Weber $1986: 243-260$; Terret 2005.

17 Roger 1978 : 229. 
persistent à conserver leur laideur», écrit Jules Claretie en $1898^{18}$. L'automobile est soupçonnée d'être la source d'un délitement de la communauté et de ses valeurs, et notamment de la place des femmes en société, car l'on s'inquiète que l'engin soit parfaitement inadapté à l'élégance féminine. Selon Octave Uzanne, homme de lettres et journaliste qui s'est intéressé aux mœurs féminines, le costume d'automobiliste, et plus généralement celui de la sportive, témoigne du fait que la femme sort de la sphère qui est la sienne: "Tout sport devient, pour la Parisienne contemporaine, un prétexte plausible à travestissements plutôt qu'il n'est une vocation physique : supprimez l'amazone, adieu le cheval. Retirez le costume spécial à l'automobiliste, à l'aéroplaneuse qui est en formation, à la bicycliste, à la chasseresse, à l'escrimeuse, et les sports féminins auront vécu $»^{19}$. Tout n'est donc pour la femme qu'une question de mode et d'apparence, puisque la parure trahit la nature. Dans le discours de la mondanité, la frivolité est une chose grave, l'apparence recouvre l'essence, d'où la frontière mince qui sépare la conformité de la déformation. L'automobile est bien, par exemple pour le chroniqueur des élégances dans le magazine Je sais tout, au début du XXe siècle, un « sport inélégant $»^{20}$; la femme doit porter de grosses lunettes et des vêtements de tissus épais et sans grâce : «Vous aviez une créature délicieuse il y a quelques minutes; vous avez maintenant devant vous un monstre auquel on ne saurait assigner ni sexe, ni âge $»^{21}$.

\section{Nouveau regard sur la « femme virile »}

On pourrait aisément multiplier les citations sur ce qu'Émile Yvon, dans le Courrier français du 19 mai 1889, dénonçait comme « la fin d'un sexe $»^{22}$. Mais il est sans doute plus intéressant de chercher à voir comment cette topique semble quelque peu se dénouer au début du $\mathrm{XX}^{\mathrm{e}}$ siècle. Le moment correspond à celui où la formule de la vieille revue mondaine va progressivement se fondre dans celle, plus

\footnotetext{
18 Claretie 1899 : 308.

19 Uzanne $1910: 334-335$.

20 «L'élégance dans les sports », Je sais tout, mai 1905.

21 «L'élégance dans les sports ", Je sais tout, mai 1905.

22 Cité par Angenot 1989: 490.
} 
moderne et mieux stabilisée, du magazine féminin. On peut dès lors proposer que le discours crépusculaire sur la femme masculinisée a fonctionné comme une sorte de relais : à partir de 1900, certains périodiques passent de l'expression de l'angoisse de la masculinisation à une forme de revendication qui intègre des éléments tirés de la sphère traditionnellement masculine. La revue Femina, publication bimensuelle illustrée créée par l'éditeur Pierre Lafitte en février 1901, et la revue La Vie heureuse, mensuel lancé par la Librairie Hachette le 15 octobre 1902, sont tout à fait exemplaires de cette tendance ${ }^{23}$. Peu analysées depuis l'étude d'Evelyne Sullerot parue en 196324, elles mériteraient d'être réexaminées. Certains autres titres pourraient accompagner cette exploration, tel qu'un magazine comme Madame et Monsieur, hebdomadaire publié entre 1905 et 1912 qui s'engage, sans succès durable toutefois, dans l'originale aventure de la conciliation des intérêts masculins et féminins dans le domaine de la mode et des divertissements. Ces publications assez luxueuses, à la mise en page aérée, abandonnant la gravure au profit de la photographie, engendrent un indéniable renouvellement des vieilles revues mondaines.

La rupture est dans la tonalité générale qu'elles adoptent vis-à-vis des femmes et de leurs occupations. S'il n'est pas exactement militant, le ton est résolument décomplexé quand il s'agit, comme l'écrit La Vie heureuse en mai 1903, de soutenir «l'initiative féminine ». Celle-ci peut être notamment littéraire et d'ailleurs, les deux magazines lancent la même année, 1904, des prix littéraires destinés aux femmes - le plus fameux étant bien sûr le prix Femina ${ }^{25}$. Ces magazines parviennent à trouver un équilibre entre les représentations de la ménagère et de la femme plus émancipée, dépassant l'opposition traditionnelle qui prévalait entre ces deux pôles. Si on peut évoquer une certaine "illisibilité culturelle» de cette formule éditoriale pour les lecteurs contemporains, selon l'expression de Mary Louise Roberts $^{26}$ à propos du quotidien féministe La Fronde, il s'agit là

\footnotetext{
23 Pour un bref historique des deux titres, voir Ducas 2003.

24 Sullerot 1963.

25 Cosnier 2009.

26 Roberts 1997.
} 
néanmoins d'une posture résolument moderne qui sera celui de bien des revues au $\mathrm{XX}^{\mathrm{e}}$ siècle, cherchant à capter l'intérêt d'un lectorat féminin plus étendu. Ainsi, entre féminin et féminisme, un équilibre est à trouver. En septembre 1910, La Vie heureuse rappelle ainsi ce qu'il faut entendre par

le bon féminisme, celui qui n'ébranle pas la constitution de la famille, qui, en faisant des professionnelles de celles qui en ont le goût ou le besoin, laisse subsister l'épouse, la mère, la maitresse de maison, la mondaine même, le féminisme enfin qui virilise, ce qui est une force, et ne masculinise pas, ce qui serait une disgrâce ${ }^{27}$.

Sans renier la nature fondamentale des deux sexes, La Vie heureuse autorise un certain croisement entre les sphères masculine et féminine. Pour sa part, Femina l'expose explicitement dès son premier numéro: "dissipons les équivoques: il ne s'agit point ici de "féminisme" ou "d'émancipation sociale" : nous laissons à d'autres le soin de masculiniser la femme et de lui enlever son charme exquis $»^{28}$. Si perdure dans ces extraits la réprobation de la masculinisation perçue comme faute grave, notamment au plan esthétique, une forme de virilité, de force conquérante, est revendiquée. À la lecture de ces magazines, on note que l'angoisse de la masculinisation n'est plus livrée sur le ton de l'effarement qui prévalait quelques années plus tôt ; elle est même, dans bien des cas, parfaitement assumée à travers l'appropriation par les femmes de nouvelles pratiques.

La représentation des sports demeure un excellent baromètre pour mesurer les sensibilités face aux diverses pratiques féminines, et il est frappant de constater à quel point des magazines comme Femina et $\mathrm{La}$ Vie heureuse s'ingénient à mettre constamment en scène les exploits sportifs réalisés par des femmes. On perçoit cette évolution tout particulièrement autour de la question de l'automobile. Les journaux féminins travaillent en effet littéralement à la rédemption de la chauffeuse. Femina constate par exemple qu'

il y a des femmes élégantes, et très élégantes même, qui font de l'automobile, et elles restent femmes, c'est-à-dire êtres de grâce et de charme, même sous ces vêtements en général grossiers et lourds, mais

27 Cité par Ducas 2003: 49.

28 Femina, 1er février 1901. 
auxquels l'art de la coquetterie qui leur est inné ajoute et crée une élégance perpétuelle ${ }^{29}$.

En apparence très convenu, ce passage masque en réalité une tactique très courante dans ces revues : la revendication s'énonce sous condition : faire de l'automobile c'est bien, mais il faut savoir rester femme. Les magazines proposent ainsi des tenues appropriées. «Naturellement, c'est la coupe large et simple qui domine: [...] elle assure aux vêtements une indiscutable originalité, sans pour cela enlever tout caractère féminin $»^{30}$, commente toujours Femina. «On est parvenu à donner de l'aisance, même de la grâce à ces vêtements spéciaux $»^{31}$, estime pour sa part un chroniqueur de Madame et Monsieur. En octobre 1903, La Vie heureuse consacre une grande double page décomplexée sur «Les femmes et l'automobilisme». Mais c'est bien Femina qui adopte le ton le plus résolu ; «Habillezvous donc en chauffeuses, mesdames et faites de l'automobile ", tonne une chroniqueuse en juillet $1902^{32}$, qui ajoute que « la véritable élégance consiste dans l'adaptation de la mise au milieu et aux circonstances », et qui appelle ainsi les femmes à sortir des carcans de l'élégance. Au moment où naissent les grandes courses cyclistes et automobiles - le tour de France naît sous l'impulsion de L'Auto-Vélo en 1903 - Femina souligne les performances féminines. En été 1901, le magazine de Pierre Lafitte suit la course qui relie Berlin à Paris :

[Au moment du départ], ce sont surtout les femmes qui attirent l'attention, qui excitent la curiosité. C'est la première fois, à Paris, en effet - car déjà cela s'est vu à Nice - que des « sportswomen » prennent part à un raid automobile et s'apprêtent à faire de jolies vitesses sur un long ruban de chemin. Voici la baronne de Zuylen, la femme du président de l'A.C.F.: elle tient le volant de direction avec autant d'autorité que le plus habile professionnel. Voici Mme Gobonr, en vrai uniforme de chauffeuse, qui conduit elle-même aussi, sa voiture et qui, dans un sourire de jolie conviction annonce qu'elle sera à Reims à midi, car elle est attendue pour déjeuner ${ }^{33}$.

\footnotetext{
29 Femina, $1^{\text {er }}$ août 1901.

30 Femina, 1 ${ }^{\text {er }}$ août 1901.

31 Madame et Monsieur, 2 juillet 1905.

32 Milady, «Les chauffeuses de Paris-Vienne », Femina, 15 juillet 1902.

33 Star., « De Paris à Berlin en automobile », Femina, 15 juillet 1901.
} 
Un texte particulièrement frappant à cet égard est signé deux ans plus tard, en juin 1903, par une certaine Smilis. L'article, intitulé «Les chauffeuses dans Paris-Madrid », mériterait d'être cité tout entier - je n'en donnerai que deux extraits. Construit en deux temps, il donne d'abord une description de la voiture qui se lance à l'assaut de la route :

La voiture gronde sourdement; une trépidation convulsive la secoue toute, elle semble impatiente; tout à coup une pédale que l'on touche, un levier que l'on manie, d'un bond sec elle se précipite vers l'espace. Le bruit du moteur se fait plus doux, il semble que le mouvement ait apaisé sa fièvre de tout à l'heure; déjà la route fuit sous les roues; de chaque côté les arbres filent avec une vitesse de cauchemar, derrière l'auto, la poussière s'élève en un nuage qui tourbillonne. [...] Une descente maintenant, la voiture s'y précipite, la poussière devient plus menaçante, elle envahit les moindres recoins, étend une lourde couche blanche sur les vêtements, le vent fouette les visages avec rage. Et toujours le bruit du moteur régulier, entêté, toujours les coups de trompe.

Alors que le reportage s'ouvre sur la puissance de la machine et que la chroniqueuse esquive la description de la femme, la féminité est pourtant subtilement réinvestie ailleurs : la voiture est en effet présentée comme cette créature un peu mystérieuse et érotisée, traversée d'une «trépidation convulsive », «impatiente » à goûter la vitesse. Il faut la «toucher» et la «manier», presque la caresser pour la faire fonctionner. Un réseau de substantifs féminins - la voiture, la route, la vitesse, les roues, la descente - accompagnés de plusieurs pronoms «elle», ajoutent enfin à ces évocations qui féminisent la puissance et réconcilient la machine et sa chauffeuse, comme si cette dernière parvenait enfin à dompter ces éléments a priori virils que sont l'engin et sa force brute. "Libérée", presque autonome, la voiture paraît entraîner la chauffeuse vers un destin qui sera pourtant assumé. En effet, s'ensuit dans la deuxième partie de l'article un plaidoyer en faveur des « chauffeuses » :

Ce n'est pas un sport de femme, dira-t-on. C'est possible, mais une trentaine de chauffeuses sont allées ainsi par la route, à Madrid, méprisant le chemin de fer. Ce n'est pas un sport de femme, mais partout on ne croise plus qu'automobiles dans lesquelles, à côté du chauffeur attentif se voient une ou deux silhouettes de femmes, encapuchonnées, enfermées dans de grands manteaux, les yeux brillants 
sous les lunettes, les narines dilatées par la griserie de l'air vif les fouettant et par le vertige délicieux de la vitesse. [...]

La femme a achevé le triomphe de l'automobile. [...] Pour celles qui n'y ont pas encore goûté, l'automobile parait encore quelque chose d'exceptionnel, plein d'inconvénients et anti-féminin ; mais les mœurs se transforment peu à peu et l'habitude les réconciliera avec l'engin nouveau et formidable ${ }^{34}$.

Ce type d'article montre comment, dans la presse mondaine et féminine, l'angoisse de la femme masculinisée s'est transformée progressivement en revendication, ou du moins laisse place aussi à l'évocation grisante d'une forme de libération. Dans des revues comme La Vie heureuse et Femina, et dans une moindre mesure Madame et Monsieur, la femme masculinisée devient la représentation d'une femme virile qui assume sa part d'émancipation ; c'est la femme auteur - souvent évoquée dans les deux revues -, l'actrice, la femme moderne qui crée quelque chose, qui (se) réalise, autant de modèles que ces magazines présentent régulièrement. Ces publications se pensent comme des encyclopédies du talent féminin; ce sont autant des magazines féminins que des espaces de réflexion sur le féminin, sur la place de la femme dans la société. Femina et La Vie heureuse portent donc un regard général sur la femme, au croisement du divertissement, de la pédagogie et de la revendication. Leur facture moderne, bien illustrée et bien renseignée, est d'ailleurs propre à l'effervescence médiatique de l'époque; elles ne sont pas sans rappeler le magazine mensuel Je sais tout, lancé en 1905 avec beaucoup de succès par le même Lafitte et visant un lectorat familial.

\section{Le reportage $(\mathrm{au})$ féminin}

Mais un autre déplacement fondamental est opéré au cœur de ces magazines, celui de la publication de reportages, le plus souvent réalisés par des femmes, sur les femmes. Femina, tout particulièrement, a su rapidement s'approprier l'écriture de la chose vue, du reportage, appartenant traditionnellement au journalisme masculin, pour le mettre au service d'une représentation des métiers féminins ou pratiqués par des femmes. Cette évolution est nettement

34 Smilis, «Les chauffeuses dans Paris-Madrid », Femina, 15 juin 1903. 
perceptible à partir de 1903, comme elle l'est ailleurs au même moment, notamment dans le quotidien de Marguerite Durand, La Fronde, rédigé et fabriqué par des femmes. Comme l'ont montré Géraldine Mulhmann ainsi que Marie-Ève Thérenty dans son dernier livre ${ }^{35}$, le reportage est un genre qui engage le corps et l'empathie du reporter, ce qui lui permet de migrer assez aisément au cœur d'une presse qui met en avant la sensibilité et l'émotion féminine. Dans Femina et La Vie heureuse certains métiers et occupations sont explorés dans des reportages assez brefs et souvent illustrés. La lectrice peut ainsi découvrir le monde des sardinières, des ouvreuses, des «dames employées » des postes, de plusieurs œuvres de charité ou encore des femmes docteurs ${ }^{36}$. La journaliste Séverine, une des toutes premières femmes reporters et surtout la plus célèbre d'entre elles, collabore régulièrement à Femina et est souvent appelée à signer ce genre de reportages ${ }^{37}$. On remarque une tendance tout à fait semblable dans La Vie heureuse, qui fait découvrir certains petits métiers, comme «les parqueuses d'huitres », ou d'autres plus prestigieux avec «Le service des femmes dans les hôpitaux en France et à l'étranger» et les femmes internes ${ }^{38}$. En août 1903, La Vie heureuse présente une journaliste américaine, Miss Banks, adepte de ce que les Américains nomment le stunt journalism ${ }^{39}$ et dont la plus célèbre figure est alors Nellie Bly. "Miss Banks a entrepris de dépeindre toutes les conditions de la femme ${ }^{40}$, explique l'article de La Vie heureuse, en pratiquant

35 Mulhmann 2004 et Thérenty 2007.

36 Jacques Fréhel, "Chez les sardinières », Femina, 15 février 1903 ; Max Rivière, "Les ouvreuses", Femina, 1er mars 1903; Jean de l'Étoile, "Les Amis de l'ouvrière », Femina, 1er avril 1903 ; Max Rivière, "Les "Dames employées" des Postes », Femina, 15 septembre 1903 ; A.C., «Doctoresse!», Femina, 15 avril 1903.

37 «Sœur Candide et son Oeuvre», Femina, 1er février 1903 ; «Une visite à l'orphelinat des arts », 15 mai 1903; «Modernes Sabines. Les femmes et le Congrès de la Paix », Femina, $1^{\text {er }}$ octobre 1903.

38 "Les parqueuses d'huitres », La Vie heureuse, novembre 1903; "Le service des femmes dans les hôpitaux en France et à l'étranger », La Vie heureuse, juin 1903 ; «Femmes internes. Hôpitaux et asiles », La Vie heureuse, janvier 1905 (les articles de La Vie heureuse sont toujours anonymes).

39 Ou « journalisme d'immersion », voir Mulhmann 2004 : 58-74.

40 «Miss Banks, une journaliste à Transformations », La Vie heureuse, août 1903. 
elle-même certains petits métiers féminins et en se mêlant aux femmes ouvrières, pour ensuite témoigner de son expérience. Les deux magazines accordent enfin une très grande place aux reportages sur les femmes artistes ${ }^{41}$. La pratique de l'interview et la visite à l'écrivaine (j'insiste sur cette féminisation du terme, courant au Québec, pour bien marquer l'opposition avec la vieille tradition de la « visite au grand écrivain $»^{42}$ ) s'impose avec force à partir de cette même année 1903.

Ainsi se développe grâce au reportage la représentation d'une féminité active, souvent professionnelle, au cœur de journaux qui avaient traditionnellement pour mission, bien au contraire, d'occulter ce genre d'activités jugées peu féminines. Il y a là une rupture assez nette qui montre que la crise de la masculinisation semble être surmontée au début du $\mathrm{XX}^{\mathrm{e}}$ siècle et que désormais l'imaginaire mondain dont continuent de se réclamer ces nouveaux magazines n'est pas incompatible avec un élargissement de la sphère des activités pratiquées par les femmes.

Précisons toutefois que cet élargissement ne s'effectue pas sans heurt entre les différents textes qui composent les magazines. Il est intéressant de voir que Femina demeure marqué par une certaine discordance éditoriale interne, notamment par des effets de reliefs idéologiques qui se dessinent dans chaque numéro entre différents espaces ou rubriques. Ainsi durant l'année 1903, alors qu'au cœur du magazine la lectrice peut découvrir les reportages sur les métiers féminins, la première page est souvent consacrée à une chronique ou à un petit conte dont la rédaction est confiée à un homme. Jules Claretie, célèbre chroniqueur au Temps, les romanciers Fernand

41 Dans Femina: Gabrielle Réval, "Les écoles des femmes-peintres », 15 février 1903 ; Maurice Guillemot sur «Mme Jean Bertheroy», 15 avril 1902 ; S.n., "Madame Séverine », 1 $1^{\text {er }}$ octobre 1902; Maurice Guillemot, "Gyp », 15 novembre 1902 ; Renée Allard, «Mme Alphonse Daudet», 15 janvier 1903 ; Séverine, "Une grande dame de lettres: Mme G. de Montgomery ", 15 avril 1903. Dans La Vie heureuse: "La plus jeune des femmes de lettres. Gérard d'Houville et "L'Inconstante" », mai 1903 ; «En visite chez Marcelle Tinayre. Sa maison, ses romans », juin 1903; "Chez Claude Ferval. La baronne Aimery de Pierrebourg », août 1903 ; «Les femmes peintres », janvier 1905.

42 Nora 1992. 
Vandérem, Abel Hermant ou encore François de Nion, tous praticiens du roman de mœurs mondaines, sont des collaborateurs assez réguliers du magazine. En faisant appel à des plumes masculines, Femina s'adjuge la collaboration d'écrivains reconnus mais aussi dépositaires d'une rhétorique mondaine très convenue : le ton charmant de Monsieur s'adressant à Madame, évoquant bals, amours secrètes de jeunes filles expiées dans le mariage, devoirs de la mondaine ... Tout cela alors que les pages voisines tendent à montrer une autre "féminité»: la femme moderne qui voyage, s'éduque, travaille, crée. D’un côté, le chroniqueur et le conteur demeurent cantonnés dans un journalisme désormais daté, d'opinion et de fiction mondaine, tandis que de l'autre les reportages souvent réalisés par des femmes plongent la lectrice au cœur des réalisations de «la femme moderne ». Femina ménage ainsi des espaces compensateurs qui « rachètent» en quelque sorte les audaces de la femme émancipée. Mais à travers le rapprochement de ces différents espaces, qui épousent poétiquement des formes liées tantôt à un journalisme plus ancien et plus littéraire, tantôt à un journalisme plus moderne et exploratoire, on peut percevoir une forme subtile de revendication. La proximité de ces zones discordantes dans une même parution provoque en effet une sorte de renversement: c'est le chroniqueur qui paraît désormais figé dans un genre et, si j'ose dire, dans sa «nature» de vieux chroniqueur, alors que le reportage au/sur le féminin s'emploie à moderniser l'image de «la femme». Les rôles traditionnels s'inversent: l'homme fabule tandis que la femme reporter fouille le réel.

D’une manière plus pragmatique enfin, remarquons que la mission généraliste du magazine de Pierre Lafitte cherche ici à coïncider avec un objectif commercial. Alors que la culture médiatique induit, comme l'a montré Dominique Kalifa ${ }^{43}$, des segmentations et des hiérarchisations de lectorat, le mouvement se joue aussi en faveur d'un élargissement des références ; le succès commercial suppose de diviser l'espace du périodique en régions complémentaires - au risque de la discordance - afin de rejoindre un plus large lectorat. Il reste toutefois indéniable qu'à l'orée $\mathrm{du} \mathrm{XX}^{\mathrm{e}}$ siècle, une nouvelle

43 Kalifa 2000. 
représentation de «la femme» travaille à modifier en profondeur la formule ancienne des publications féminines et mondaines. Une recherche complémentaire sur l'usage de la photographie dans Femina et $\mathrm{La}$ Vie heureuse pourrait aussi montrer qu'une forme de "réalisme » s'impose dans les multiples représentations visuelles des femmes, rendant obsolète la vieille sémiotique de la gravure qui enfermait elle aussi leur image dans un cadre inamovible.

La représentation de «la femme» dans les journaux fonctionne comme un marqueur des diverses étapes idéologiques que traverse la presse tout au long du XIXe siècle. Au temps de la presse d'opinion - laquelle domine l'essentiel du siècle - «la femme» est un objet manipulé idéologiquement, pris en charge par un discours dominant. Mais, avec la naissance de la presse d'information, de nouveaux genres - notamment l'interview et le reportage - tendent à ménager des discours alternatifs au cœur de nouvelles expériences médiatiques, comme au sein des magazines féminins modernes. La « crise» de la masculinisation se déroule ainsi significativement au moment où la presse est en train de redécouper les frontières poétiques, génériques et sexuées qui sont les siennes. De la femme masculinisée au reporter féminisé, de la topique imposée à la revendication par l'appropriation féminine de certaines pratiques liées au genre masculin, se mesure une évolution qui montre que l'historicité de la presse est directement liée à ces variations socio-poétiques. Des revues comme Femina et La Vie heureuse, en proposant un imaginaire féminin modernisé contribuent dans une certaine mesure, à désamorcer la peur de la masculinisation des femmes.

\section{Bibliographie}

ANGENOT Marc, 1989, 1889, un état du discours social, Longueil, Le Préambule (coll. «L'Univers des discours »).

BLum Françoise, 2002, «Revues féminines, revues féministes », in Jacqueline PLUETDespatin, Michel Leymarie \& Jean-Yves Mollier (dir.), La Belle Époque des revues 1880-1914, Paris, Éditions de l'Imec, p. 211-222.

Bourdieu Pierre, 1998, La domination masculine, Paris, Seuil (coll. « Liber »). 
Castelbajac Constance de, 2003, Journal de Constance de Castelbajac, marquise de Breteuil, 1885-1886, Paris, Perrin.

Claretie Jules, 1899, La Vie à Paris 1898, Paris, Charpentier et Fasquelle.

Cosnier Colette, 2009, Les dames de Fémina. Un féminisme mystifié, Rennes, Presses universitaires de Rennes (coll. «Archives du féminisme»).

DuCAS Sylvie, 2003, "Le prix Femina: la consécration littéraire au féminin », Recherches féministes, 16/1, p. 43-95.

Eveno Patrick, 2003, «Les médias sont-ils sexués? Éléments pour une "gender history" des médias français ", Le Temps des médias, 1, p. 162-173.

Flamant-PAPARATTI Danielle, 1984, Bien-pensantes, cocodettes et bas-bleus. La femme bourgeoise à travers la presse féminine et familiale (1873-1887), Paris, Denoël.

Kalifa Dominique, 2000, «L'entrée de la France en régime médiatique : l'étape des années 1860 », in Jacques Migozzi (dir.), De l'écrit à l'écran, Limoges, Presses Universitaires de Limoges, p. 39-51.

KLEJMAN Laurence \& ROCHEFORT Florence, 1989, L'Égalité en marche. Le féminisme sous la Troisième République, Paris, Presses de la Fondation nationale des sciences politiques.

Langlois Pamela, 1979, The Feminist Press in England and France, 1875-1900, Université du Massachussets, Amherst, thèse de doctorat.

Mulhmann Géraldine, 2004, Une histoire politique du journalisme. XIX-XXe siècle, Paris, Presses universitaires de France (coll. « Partage du savoir »).

Murat Laure, 2006, La loi du genre. Une histoire culturelle du "troisième sexe», Paris, Fayard.

Perrot Michelle, 1998, Les femmes ou les silences de l'histoire, Paris, Flammarion (coll. « Champs »).

PInÇON Michel \& Monique PinÇON-ChARlot, 1995, «Beaux quartiers et stations balnéaires : la logique de l'entre-soi menacée », in Claude-Isabelle BRELOT (dir.), Noblesses et villes (1789-1950). Actes du colloque de Tours (17-19 mars 1994), Université de Tours, Maison des Sciences de la ville, p. 205-214.

PInSON Guillaume, 2008, Fiction du monde. De la presse mondaine à la Marcel Proust, Montréal, Presses de l’Université de Montréal (coll. «Socius »).

Planté Christine, 1989, La Petite scur de Balzac. Essai sur la femme auteur, Paris, Seuil (coll. «Libres à elles »).

ROBERTS Mary Louise, 1997, «Copie subversive : Le journalisme féministe en France à la fin du siècle dernier », CLIO Histoire, Femmes et Société, 6, p. 230-247. 
ROCHEFORT Florence, 1999, «L'antiféminisme à la Belle Époque une rhétorique réactionnaire ", in Christine BARD (dir.), Un siècle d'antiféminisme, Paris, Fayard, p. 133-147.

Roger Alain, 1978, Nus et paysages. Essai sur la fonction de l'art, Paris, Aubier.

Sullerot Evelyne, 1963, La presse féminine, Paris, Armand Colin.

Terret Thierry (dir.), 2005, Sport et genre, Paris, l'Harmattan.

THÉRENTY Marie-Ève, 2007, La littérature an quotidien, Paris, Seuil.

NORA Olivier, 1992, «La visite au grand écrivain », in Pierre NORA (dir.), Les lieux de mémoire, t. II, 3, Paris, Gallimard, p. 563-587.

Uzanne Octave, 1910, Parisiennes de ce temps, en leurs divers milieux, états et conditions, Paris, Mercure de France.

WeBER Eugen, 1986, Fin de siècle, Paris, Fayard. 